\title{
Thermoelastic stress analysis of titanium biomedical spinal cages printed in 3D
}

\author{
by A. Quattrocchi*, D. Palumbo**, D. Alizzio*, U. Galietti** and R. Montanini* \\ * University of Messina, Department of Engineering, 98166, Messina, Italy, \\ \{antonino.quattrocchi/damiano.alizzio/roberto.montanini\}@unime.it \\ ** Polytechnic University of Bari, Department of Mechanics, Mathematics and Management, 70125, Bari, Italy, \\ \{davide.palumbo/unberto.galietti\}@poliba.it
}

\begin{abstract}
Transforaminal lumbar interbody fusion (TLIF) cages are employed in lumbosacral spine surgery. These implants are made in Titanium and offer biocompatibility, high resistance to corrosion and a good mechanical performance. In the last years, Additive Manufacturing (AM) has been introduced in many biomedical applications, thanks to the possibility of improving complex geometry and customization for specific patient. In this regard, compensation of real mechanical behaviour is a critical issue to be investigated. The present work is focused on the evaluation of stress distribution for a 3D printed TLIF cage by means of non-contact measurements as Thermoelastic Stress Analysis (TSA). A suitable setup has been adopted to guarantee the correct loading application and an optical access to the sample.
\end{abstract}

\section{Introduction}

Titanium and its alloys are largely used in biomedical applications thanks to their high value of the strength to density ratio, high resistance to corrosion and biocompatibility. In the last years, additive manufacturing (AM) has made it possible to obtain titanium medical components with a complex geometry. Furthermore, having a limited production, these types of implants can be produced in a relative short time, with a better mechanical quality and, above all, customized for the specific patient's needs. A typical use of AM in biomedical applications is represented by transforaminal lumbar interbody fusion (TLIF) cages. These prostheses are employed to stabilize lumbosacral spine diseases and promote intervertebral fusion. Their design is finalized to improve physiologic movement of the body, reducing over mobility of spinal segment and favouring shock absorption capability [1]. In this field of interest, a proper mechanical behaviour is a critical issue. As a matter of fact, reliability of any prosthesis has to be guaranteed in terms of structural integrity, adequate stress propagation and limited strain condition. AM represents a further parameter to be taken into consideration, since it determines different properties with respect to the traditional manufacturing process. Considering TLIF cages small size and lattice structures properties, the above-mentioned aspects can only be investigated through non-contact techniques capable of providing information in full field. [2-4]. One drawback of optical methods is the necessity of having an optical access of the region to be examined, which can be tricky in some cases. Moreover, TLIF cages do not present parallel surfaces and for such reason a specific setup is required to apply a controlled load. Thermal stress analysis (TSA) method allows to obtain a quantitative assessment of the state of stress in a structure. By applying a cyclical load, within the elastic range of the material, and measuring the thermal response, a stress map of the sample is achieved [5]. For all metals, in particular Titanium, thermoelastic effect is more evident due to the high load in elastic range [6]. Okazaki et al. [7] employed this technique to investigate the effect of stress concentration on durability of various orthopaedic implant devices. Hyodo et al. [8] performed tests on both synthetic intact femurs and implanted femurs with an artificial joint. In this case, results show that TSA can be used for predicting pre-clinical performance of new artificial joint design.

The aim of this work is to investigate the mechanical behaviour of TLIF cages in Titanium lattice structure by means TSA technique. A dedicated setup has been established to apply a controlled load to the implant and perform correct optical measurements. Different types of test have been carried out to optimize investigation parameters and define stress map distribution.

\section{Theory of Thermoelastic Stress Analysis}

Thermoelastic stress analysis allows the quantitative assessment of the state of stress in a structure through the measurement of the thermal response resulting from the application of a load within the elastic range of the material [6], [7]. This approach assumes the material properties constant with temperature, which is demonstrated not to be correct for all materials.

Thermoelastic theory, and subsequent experimental results, has already shown that the thermographic signal is also a function of the mean stress and not only of the mechanical properties of the material. In particular, it depends on the variation of longitudinal modulus of elasticity with temperature [6-7], [10].

For an isotropic material without any internal heat source, in linear-elastic and adiabatic conditions, the temperature variations can be described by the following equation [5]: 


$$
\rho C_{\varepsilon} \frac{\dot{T}}{T}=-\left[\alpha+\left(\frac{v}{E^{2}} \frac{\partial E}{\partial T}-\frac{1}{E} \frac{\partial v}{\partial T}\right) s\right] \dot{S}+\left[\frac{(1+v)}{E^{2}} \frac{\partial E}{\partial T}-\frac{1}{E} \frac{\partial v}{\partial T}\right] \sigma_{i} \dot{\sigma}_{i}
$$

where $\rho$ is the density of the material, $C_{\varepsilon}$ is the specific heat under constant strain, $T$ is the temperature, $\sigma_{i}$ is the principal stress (summing over $i, j$ with $i, j=1-3$ ), $\alpha$ is the coefficient of linear expansion, $v$ is the Poisson ratio, $E$ is the Young modulus and $s$ is the first stress invariant. The dotted symbols represent derivatives with respect to the time. In a similar way to the works of Palumbo et al. [5], two material constants can be defined as reported in Equation (2):

$$
a=\frac{\alpha}{\rho C_{\varepsilon}}, \quad b=\frac{1}{E^{2}} \frac{\partial E}{\partial T} \frac{1}{\rho C_{\varepsilon}}
$$

Substituting Eq. (2) in Eq. (1) and neglecting the variations of the Poisson's ratio with the temperature [9], we obtain:

$$
\frac{\dot{T}}{T}=-[a+v b s] \dot{s}+(b+v b) \sum_{i} \sigma_{i} \dot{\sigma}_{i}=-a \dot{s}+b\left[v s \dot{s}-(1+v) \sum_{i} \sigma_{i} \dot{\sigma}_{i}\right]
$$

The second member in Eq. (3) represents the contribution of the second-order effects on temperature variations. This contribution depends on the material properties and stress conditions. As an example, for some materials such as Titanium, the second-order effects can be significant, above all in the presence of high-stress values. In these conditions, Eq. (3) should be used for relating the temperature variations and the stresses.

If the second-order effects are not considered, we obtain the classic TSA equation from which the sum of the principal stresses can be evaluated through the thermoelastic constant $a$ :

$$
\frac{\dot{T}}{T}=-a \dot{S}
$$

\section{Materials and Methods}

\subsection{TLIF cage}

The analysed implant is a commercial TLIF cage (Figure 1), made of a medical grade Titanium by means of electron beam melting (EBM). Its design is characterized by a full body, on which there is a socket, and by four faces, that show a trabecular structure. The socket is used to connect a specific medical tool, that facilitates the correct positioning of the TLIF cage. These different parts work with the aim of recreating and maintaining the distance between the vertebras, promoting biological fusion and supporting the physiological load in the lumbosacral spine.

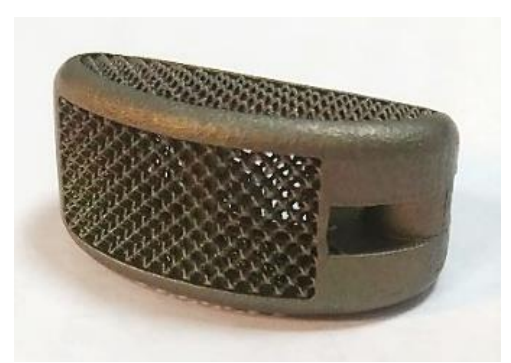

a)

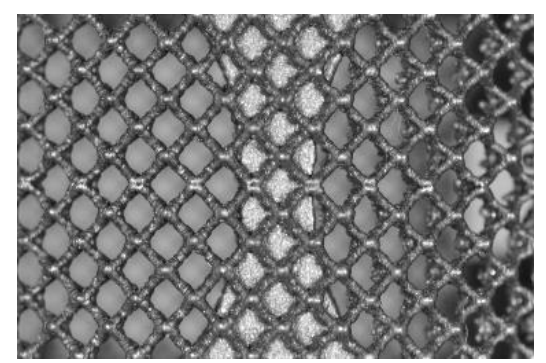

b)

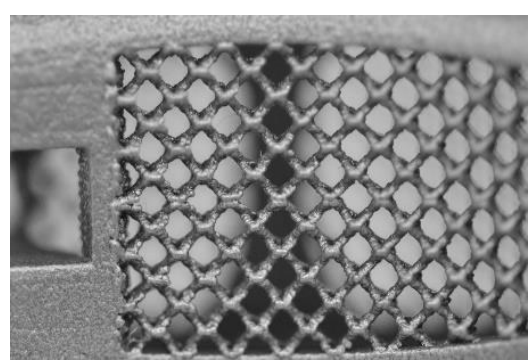

C)

Fig. 1: View of the structure of the TLIF cage: a) full view, b) front trabeculae view, c) rear trabeculae view. The images are not in scale.

The sizes of the TLIF cage (Table 1) are generally limited, although they depend on the severity of the individual patient's surgery and can be increased for specific applications. In this application, a TLIF cage of $26.00 \mathrm{~mm} \times 9.00 \mathrm{~mm} \times$ $13.00 \mathrm{~mm} \times 5^{\circ}$ (length $\times$ width $\times$ height $\times$ angle) was analysed. The elementary unit of the lattice structure is a cubic cell of $1.25 \mathrm{~mm}$, which develops in the $\mathrm{x}-, \mathrm{y}-$, and $\mathrm{z}$-directions on a single plane. 
Table 1: Mechanical and thermo-physical properties of the considered material.

\begin{tabular}{|c|c|c|c|c|c|c|c|c|c|}
\hline Material & $\alpha\left(\mathrm{K}^{-1}\right)$ & $\rho\left(\mathrm{Kg} / \mathrm{m}^{3}\right)$ & $\begin{array}{c}C_{p^{1}} \\
(\mathrm{~J} / \mathrm{KgK})\end{array}$ & $\begin{array}{c}C_{\varepsilon}{ }^{2} \\
(\mathrm{~J} / \mathrm{Kg} \cdot \mathrm{K})\end{array}$ & $\begin{array}{c}E \\
(\mathrm{GPa})\end{array}$ & $\mathbf{v}$ & $\begin{array}{c}\partial E / \partial T \\
(\mathrm{MPa} / \mathrm{K})\end{array}$ & $\begin{array}{l}R_{\mathrm{p} 0.2^{3}} \\
(\mathrm{MPa})\end{array}$ & $\begin{array}{c}b / a \\
\left(\mathrm{MPa}^{-1}\right)\end{array}$ \\
\hline Ti6Al4V & $8.6 \times 10^{-6}$ & $4.43 \times 10^{3}$ & 560 & 560 & 114 & 0.34 & -48 & 1100 & 4.31E-04 \\
\hline
\end{tabular}

\subsection{Experimental setup}

A dedicated setup (Figure 2) was established to allow an optical window of analysis thanks to a customized system for physiological load application. The TLIF cage was enclosed between two sample supports and subjected to a cyclic compression by means of an electromechanical material testing machine (mod. ElectroPulse E3000, Instron), equipped with two horizontal plates and a calibrated load cell. The two sample supports were specifically manufactured to ensure the correct transfer of the load and, for this reason, were made of a cobalt-chromium (CoCr) alloy using a resin casting process.

The TSA thermographic sequences were acquired by adopting an IR camera (mod. SC7600, FLIR) with a cooled InSb detector, resolution of $640 \times 512$ pixels, NETD $<20 \mathrm{mK}$ at room temperature and medium infrared range (MWIR 3.6$5.1 \mu \mathrm{m})$. The camera was equipped with a $50 \mathrm{~mm}$ lens and an extension ring of $20 \mathrm{~mm}$ and placed approximately at a distance of $40 \mathrm{~cm}$ from the specimen. The IR signal and the applied harmonic load were simultaneously synchronized (lock-in mode), stored and subsequently processed by dedicated software.

The main flaw with these measurements is that the camera must have a sufficient depth of field to adequately focus the whole elementary cell of the lattice structure. Such necessity means that, due to the constructive characteristics of the lenses, the analyzable portion of the sample is limited.

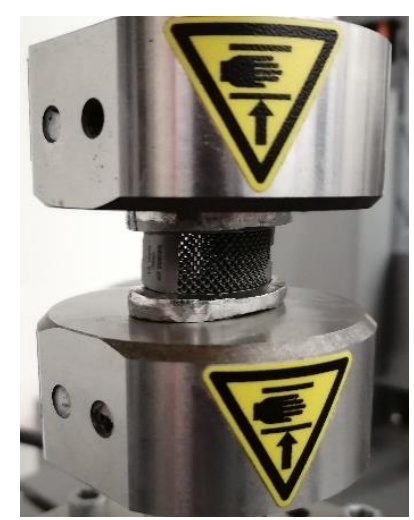

a)

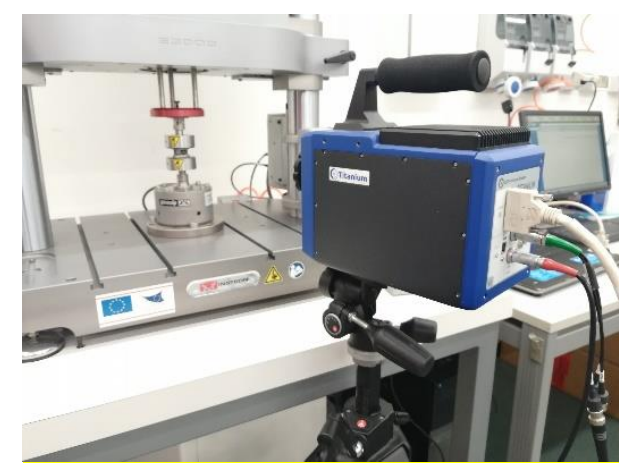

b)

Fig. 2: a) stress transfer system and b) experimental setup used for performing TSA tests

\subsection{Testing procedures}

All tests were performed in load control, transferring the stress from the flat plates of the electromechanical material testing machine to the TLIF cage by means of the sample supports. The repeatability of the performed measurements was assessed. Furthermore, the TLIF cage was investigated according to four different views (front, $\pm 30^{\circ}$ and rear) to overcome the technological limits of the used lenses and to ensure optimal focus of the inspected lattice structure.

TSA measurements were obtained using three specific load configurations, as shown in Table 2. The same stress loading ratio $R$ between the maximum $F_{\max }$ and minimum load $F_{\min }$, and the same load frequency $f$ were adopted for all tests, while the load amplitude $\Delta F$ was changed.

Table 2: Load configuration for TSA measurements.

\begin{tabular}{|c|c|c|c|c|c|}
\hline & $\boldsymbol{f}[\mathrm{Hz}]$ & $\boldsymbol{R}[]$ & $\boldsymbol{F}_{\max }[\mathbf{N}]$ & $\boldsymbol{\Delta}[\mathbf{N}]$ & $\boldsymbol{F}_{\min }[\mathrm{N}]$ \\
\hline Configuration I & 9.00 & 0.1 & -10 & -45 & -100 \\
\hline Configuration II & 9.00 & 0.1 & -18 & -81 & -180 \\
\hline Configuration III & 9.00 & 0.1 & -36 & -162 & -360 \\
\hline
\end{tabular}




\section{Data processing}

Thermal sequences acquired during the experimental tests were processed with the IRTA® [11] software to obtain the thermoelastic data. In particular, the temperature signal has been processed in the frequency domain by using the temperature model represented in Eq. 5:

$$
T(t)=T_{0}+T_{1} \sin \left(\omega t+\varphi_{1}\right)+T_{2} \sin \left(2 \omega t+\varphi_{2}\right)
$$

here $T_{0}$ is the mean temperature, $T_{1}, T_{2}$ and $\varphi_{1}, \varphi_{2}$ are the first and second amplitude harmonics and the phase shifts of first and second harmonics respectively, while $\omega=2 \pi f$ is the angular frequency. In this work, we focus the attention only on the first harmonic of the temperature signal $\left(T_{1}, \varphi_{1}\right)$. TSA maps were obtained applying Eq. (5) pixel by pixel.

In Figure 3 are shown two thermal maps extracted from the thermal sequence, acquired with the configuration III (first repetition) and referre to the frontal acquisition. It can be seen that many hot areas appear due to reflections of surrounding objects and of the IR camera itself. Furthermore, the central structural element presents a high roughness (typical of AM components) with the presence also of some voids. All these reflections are sources of noise that can be magnified by the motion effects during the load application. In the next section, the effect of these source of noise on TSA data will be discussed.

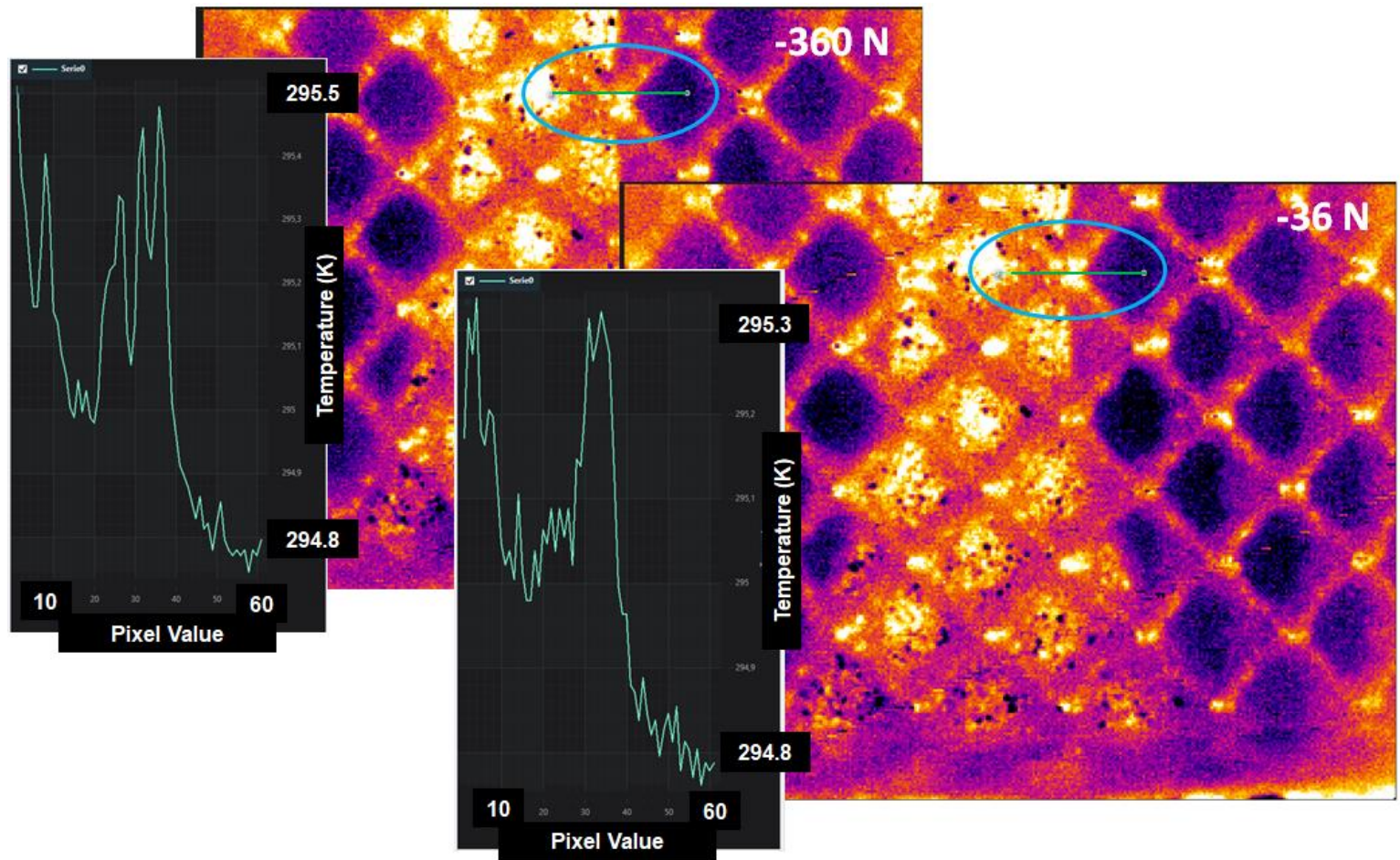

Fig. 3: Motion effects during the application of dynamic loadings: thermal frames at the maximum (-360 N) and minimum (-36 N) loads in compression (Configuration III)

\subsection{TSA data calibration procedure}

The calibration procedure allows for obtaining the thermoelastic maps in terms of stresses (first invariant). As already said in section 2, if the stresses are low and very far from the material yield stress, the Eq. (4) can be used to compute stress values. Under sinusoidal loading, integrating the Eq. (4), we obtain:

$$
\Delta s=\Delta\left(\sigma_{1}+\sigma_{2}\right)=-\frac{\Delta T}{T_{0}} \frac{1}{a}=-\frac{2 T_{1}}{T_{0}} \frac{1}{a}
$$

The thermoelastic constant $a$ has been calculated referring to the literature values reported in Table 1. $T_{1}$ and $T_{0}$ are the value of thermoelastic and temperature signal provided from the Eq. (5), respectively. 


\section{Results and Discussion}

In Figure 4, the results in terms of TSA maps are summarized. In particular, for the test configuration III and for each setup configuration, the map of the amplitude of the first invariant is reported. Moreover, to investigate the sign of the first invariant, the maps of the cosine component $\left(\Delta s^{*} \cos \varphi\right)$ are also reported.

First considerations about results regard the "hot stress" and noise areas present in each map. As already anticipated in the previous section, reflections associated with a significant motion of pixels produce spurious with false very high-stress signals. These areas cannot be considered for the analysis of the mechanical behaviour of the TLIF cage. However, the spurious hot spots can be simply identified from thermal sequences. In all the setup configurations, the false "hot stress" areas are present above all in correspondence of the trabecular knots.

The motion effect can be seen also in the repetition tests. In Figure 5, are shown the amplitude maps referred to three repetitions carried out according to the configuration I (frontal view). In the same Figure, are reported the results obtained subtracting the maps each other. In these maps, the maximum/minimum values of the first invariant are about 5 $\mathrm{MPa}$. This latter can seem significant with respect to the maximum value of stresses in the amplitude map. However, as already said, it is due to the combined effect of reflections and motion that generate a spurious stress signal. In this regard, the results show good repeatability of TSA measurements.
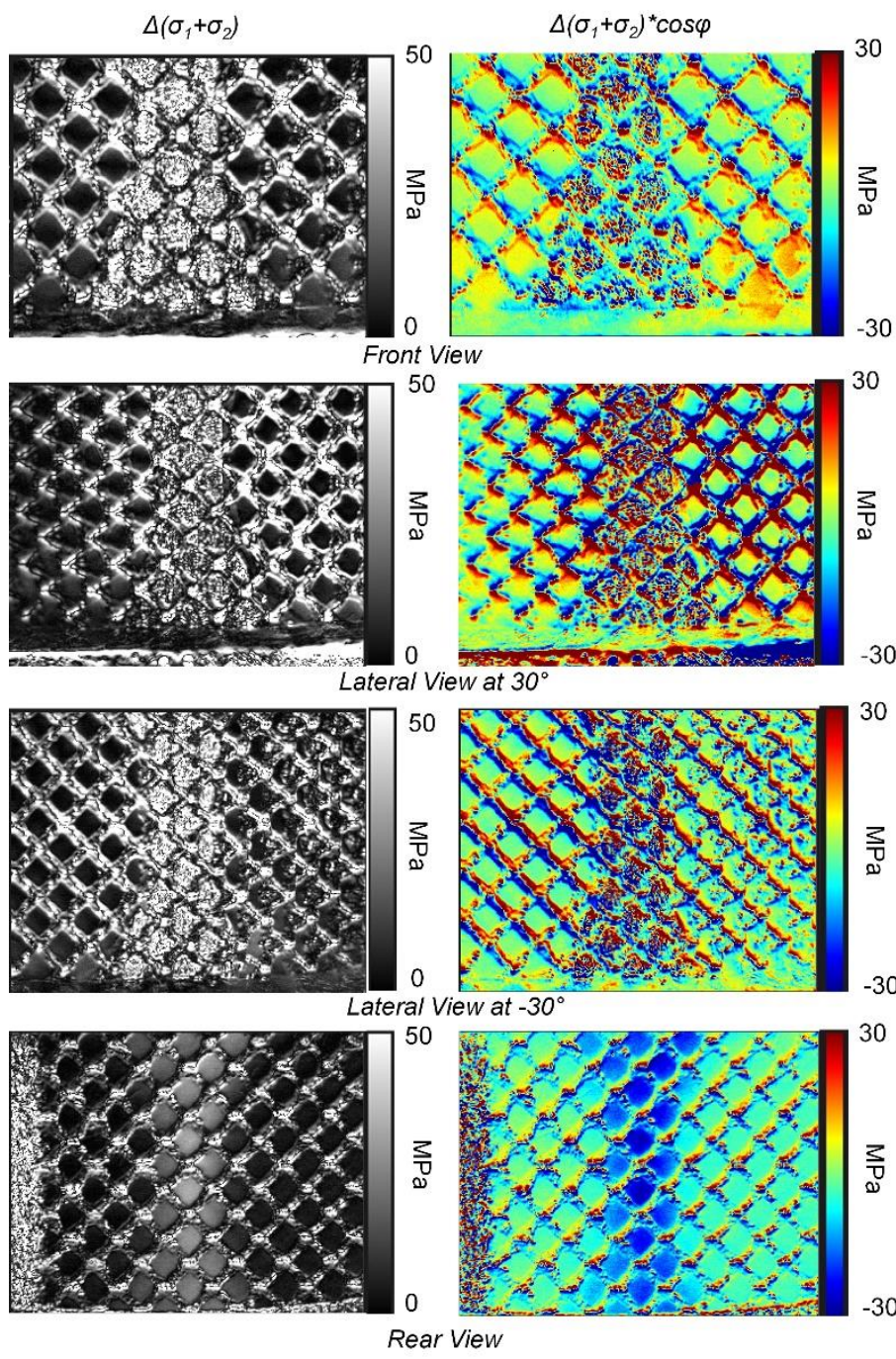

Fig. 4: Thermoelastic maps: First invariant (left) and its cosine component (right) for each test setup

All TSA maps allow for obtaining information about the mechanical behaviour of the implant subjected to compression. Firstly, the trabecular elements present a complex stress signal that seems differ point by point. This behaviour is due to the complex and irregular geometrical shape of the trabeculae. However, a common mechanical behaviour has been observed for all the configurations. Specifically, trabeculae are subjected to bending stresses that seem to increase from the top to the bottom of the TLIF cage. This behaviour appears evident in the stress maps with the sign information. In fact, it can be observed the change of the stress sign on the trabeculae structures that represents the 
typical bending behaviour on TSA maps. In this regard, it is also interesting to notice in the amplitude maps zero-stress zones, that pass through the trabecular structure and that represent stress-free zones.

Quantitative stress evaluations on the vertical structural element can be done only for the rear configuration setup. In fact, the stress signal in the front configurations is too noisy and cannot be used for quantitative evaluations. This element has the task of increasing the stiffness of the TLIF cage in the vertical direction. The map related to the rear configuration shows that the central structural element is subjected to compressive stresses that seem non-uniform on the element. To investigate more deeply the behaviour on the vertical element, three areas along its longitudinal direction were considered. The mean value of the amplitude signal in each area has been reported as a function of the amplitude applied load in each configuration (Figure 6). In particular, for each set of data the linear regression analysis has been performed. This latter shows that for each area the linear relationship between the first invariant and the applied loads. The results confirm the hypothesis of linear-elastic behaviour and the neglectable effect of mean stresses.
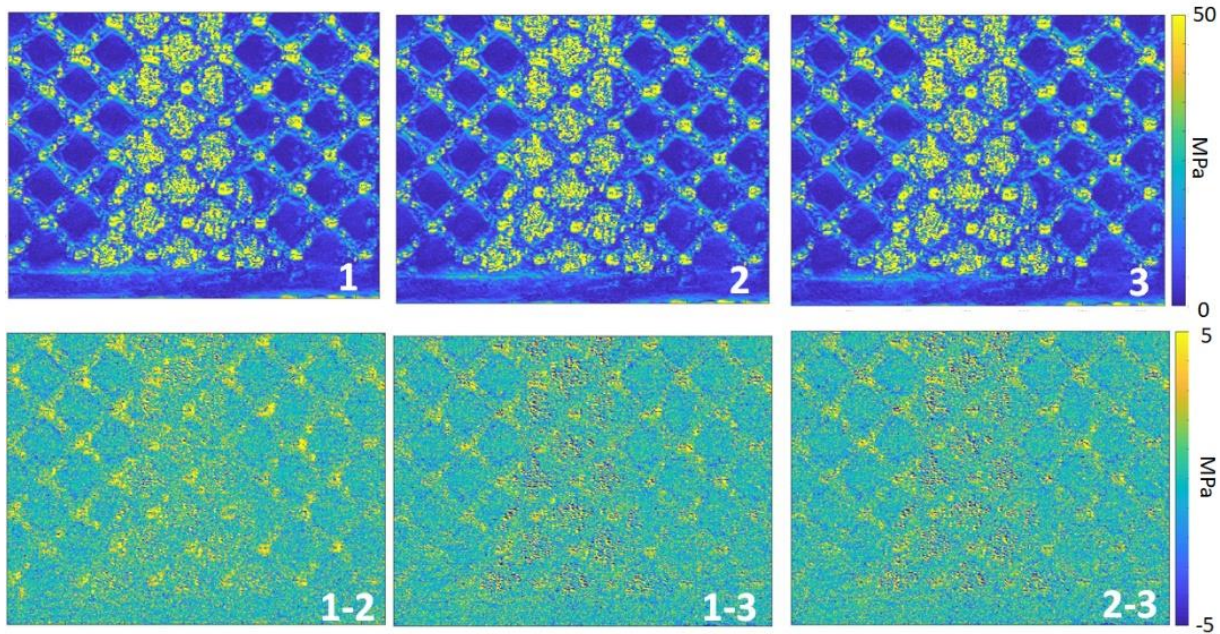

Fig. 5: Thermoelastic maps of the first invariant (front view): maps of the three replications (top) and images subtraction analysis

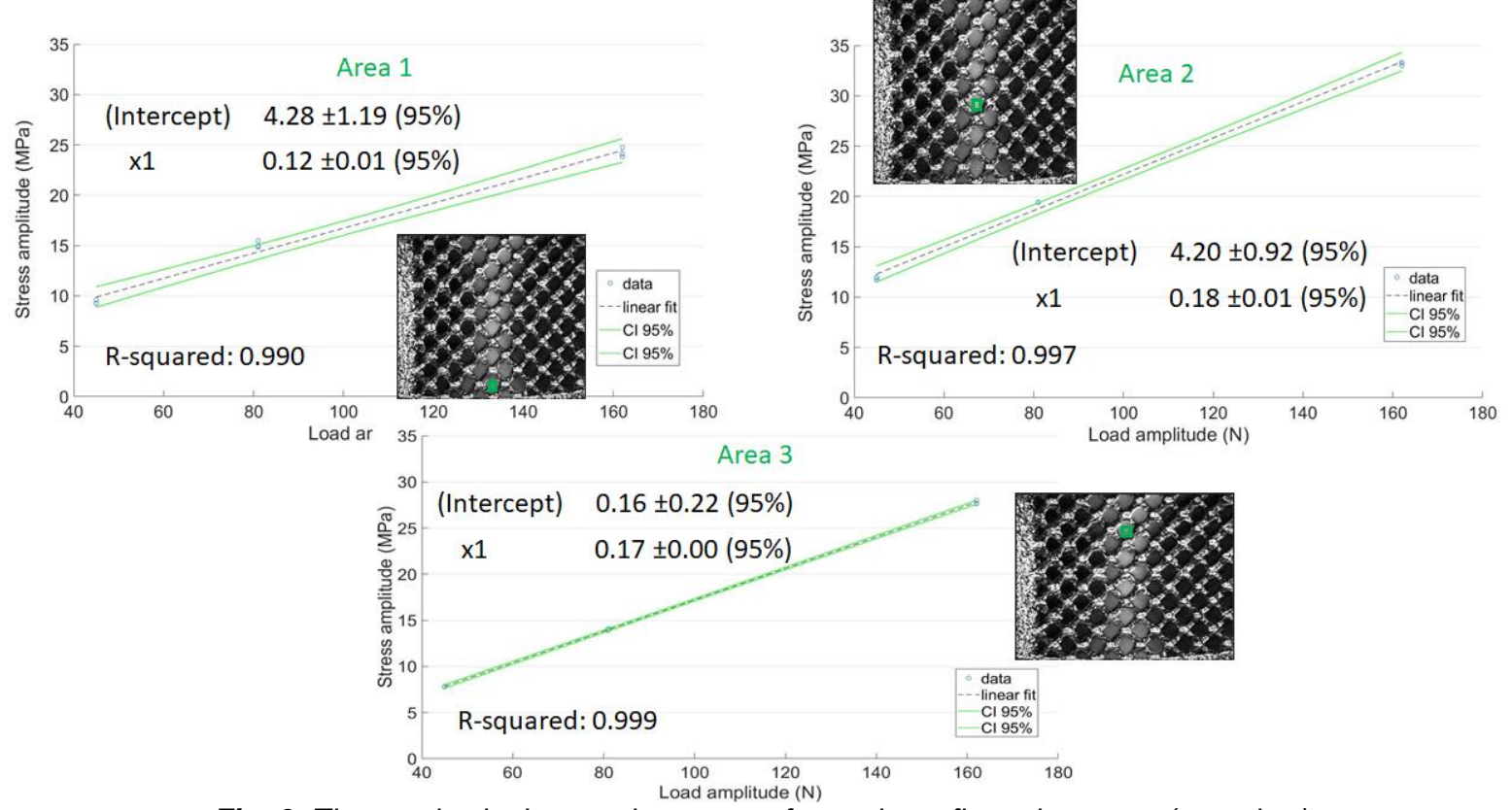

Fig. 6: Thermoelastic data on three areas for each configuration setup (rear view)

Comparing the results among the three areas, differences in the stress values can be observed. Stresses in the Area n. 2 are systematically higher that the other two areas. In particular, the difference of average stress values seems 
increasing as the applied loads increase. These results indicate the possible presence of an out of plane bending deformation that increases as the applied loads increase (buckling).

Summarizing, the TLIF cage presents a complex geometry that determines local differences in the mechanical behaviour among trabeculae elements. All these elements are subjected to bending under compressive loads and local high stress values were observed in correspondence of the trabecular knots. The vertical central element is interested to a buckling behaviour that could compromise the structural integrity at higher loads.

\section{Conclusions}

In this work, The TSA technique has been used for investigating the mechanical behaviour of a TLIF cage implant made of a medical grade Titanium by means of electron beam melting (EBM). In particular, a suitable experimental set-up has been used for subjecting the implant to dynamic compression loads by means of appropriate supports.

Different tests with different loading conditions were carried out and several TSA analyses were performed framing the implant from different views. Stress maps were obtained calibrating the temperature data by means of the thermoelastic constant. This latter was estimated considering the literature values of the material constants.

The main results can be summarized as follows:

- the TLIF cage presents a complex geometry that determines local differences in the mechanical behaviour among trabeculae elements,

- $\quad$ trabeculae elements are subjected to bending under compressive loads and local high stress values were observed in correspondence of the trabecular knots,

- the vertical central element is interested to a buckling behaviour that could compromise the structural integrity at higher loads.

Future works will be focused on the study of the mechanical behaviour using other experimental approaches such as the DIC (Digital Image Correlation) technique and on the investigation of the fatigue behaviour under dynamic compressive loads.

\section{Acknowledgments}

tests.

The authors would like to thank Mr Vito Conticchio for the manufacturing of the supports used for experimental

\section{REFERENCES}

[1] Cappuccino A., Cornwall G. B., Turner A. W., Fogel G. R., Duong H. T., Kim K. D., Brodke D. S. "Biomechanical analysis and review of lateral lumbar fusion constructs". Spine 35(26S), pp. S361-S367, 2010.

[2] Allevi G., Castellini P., Chiariotti P., Docchio F., Marsili R., Montanini R., Pasinetti S., Quattrocchi A., Rossetti R., Rossi G., Sansoni G., Tomasini E. P. "Qualification of additive manufactured trabecular structures using a multi-instrumental approach. In 2019 IEEE International Instrumentation and Measurement Technology Conference (I2MTC) pp. 1-6, 2019.

[3] Allevi G., Capponi L., Castellini P., Chiariotti P., Docchio F., Freni F., Marsili R., Martarelli M., Montanini R., Pasinetti S., Quattrocchi A., Rossetti R., Rossi G., Sansoni G., Tomasini E. P. "Investigating additive manufactured lattice structures: a multi-instrument approach". Transaction of Instrumentation and Measurements, in press, 2019

[4] Allevi G., Cibeca M., Fioretti R., Marsili R., Montanini R., Rossi, G. "Qualification of additively manufactured aerospace brackets: A comparison between thermoelastic stress analysis and theoretical results". Measurement 126, pp. 252-258, 2018.

[5] Palumbo D., Galietti U. "Data Correction for Thermoelastic Stress Analysis on Titanium Components". Experimental Mechanics 56(3), pp. 451-462, 2015.

[6] Di Carolo F., De Finis R., Palumbo D., Galietti U. "A thermoelastic stress analysis general model: Study of the influence of biaxial residual stress on aluminium and titanium". Metals 9(6) 671, 2019.

[7] Okazaki Y., Ishii D., Ogawa A. "Spatial stress distribution analysis by thermoelastic stress measurement evaluation of effect of stress concentration on durability of various orthopedic implant devices". Materials Science and Engineering C 75, pp. 34-42, 2017.

[8] Hyodo K., Inomoto M., Ma W., Miyakawa S., Tateishi T. "Thermoelastic Femoral Stress Imaging for Experimental Evaluation of Hip Prosthesis Design”. JSME International Journal 44 (4), pp. 1065-1071, 2001.

[9] M. Fukuhara, A. Sanpei, Elastic moduli and internal frictions of Inconel 718 and Ti-6Al-4V as a function of temperature, J. Mater. Sci. Lett. 12 (1993), 1112-1124.

[10] F. Di Carolo, R. De Finis, D. Palumbo, U. Galietti, Study of the thermo-elastic stress analysis (TSA) sensitivity in the evaluation of residual stress in non-ferrous metal, in Thermosense: Thermal Infrared Applications XLI 2019; Baltimore; United States; 15 April 2019 through 17 April 2019; Code 151454, Volume 11004, Article number 1100400.

[11] IRTA ver. 2.0 (2020). Diagnostic Engineering Solutions (DES srl). 